\title{
Estimativa do plastocrono em meloeiro (Cucumis melo L.) cultivado em estufa plástica em diferentes épocas do ano
}

\author{
Estimating the plastochron in muskmelon (Cucumis melo L.) grown inside plastic \\ greenhouse at different planting dates
}

\section{Nereu Augusto Streck ${ }^{1}$ Tiago Tibola ${ }^{2}$ Isabel Lago ${ }^{2}$ Galileo Adeli Buriol ${ }^{3}$ Arno Bernardo Heldwein ${ }^{4}$ Flavio Miguel Schneider ${ }^{1}$ Vivairo Zago ${ }^{5}$}

\section{RESUMO}

O meloeiro (Cucumis melo L.) é uma hortaliça de alto valor econômico. A emissão de nós é um componente importante em modelos matemáticos de simulação do crescimento e desenvolvimento de culturas de hábito de crescimento decumbente como o meloeiro e outras cucurbitáceas. A emissão de nós pode ser calculada utilizando-se o conceito do plastocrono, que é o intervalo de tempo entre o aparecimento de nós sucessivos em uma haste de dicotiledôneas. Quando a soma térmica é usada como medida de tempo fisiológico em plantas, o plastocrono tem como unidades ${ }^{\circ} \mathrm{C}$ dia nó ${ }^{-1}$. Estudos anteriores mostraram que o plastocrono em meloeiro varia com o genótipo e a época de cultivo. Este trabalho teve por objetivo estimar o plastocrono em meloeiro transplantado em diferentes épocas de cultivo no interior de estufa plástica. Foram realizadas 12 épocas de semeadura e transplante no interior de uma estufa plástica de $10 \mathrm{~m} X 25 \mathrm{~m}$, coberta com polietileno transparente de baixa densidade, localizada no Campo Experimental do Departamento de Fitotecnia da Universidade Federal de Santa Maria, RS. O híbrido utilizado foi o HY-MARK (grupo Cantaloupe). Em 12 plantas etiquetadas por época de transplante, contou-se o número de nós visíveis (NN) na haste principal da planta duas vezes por semana. A soma térmica diária (STd, ${ }^{\circ} \mathrm{C}$ dia) foi calculada levando-se em conta as temperaturas cardinais de aparecimento de nós em meloeiro (10, 34 e $\left.45{ }^{\circ} \mathrm{C}\right)$. A soma térmica acumulada (STa, ${ }^{\circ} \mathrm{C}$ dia), a partir da data de transplante das plântulas, foi calculada somando-se a STd. O plastocrono foi calculado como sendo o inverso do coeficiente angular da regressão linear entre $N N$ e STa para cada época. O plastocrono calculado variou entre as épocas de cultivo de 13,4 a $21,8^{\circ} \mathrm{C}$ dia nó ${ }^{-1}$, com um valor médio de $18,6( \pm 2,3)^{\circ} \mathrm{C}$ dia nó ${ }^{-1}$. Esta diferença de plastocrono entre as épocas de cultivo pode representar vários dias do calendário civil se o interesse é predizer eventos associados ao NN na planta de meloeiro, como por exemplo a data de abertura da primeria flor masculina e da primeria flor hermafrodita.

Palavras-chave: temperatura, soma térmica, emissão de nós, desenvolvimento vegetal.

\section{ABSTRACT}

Muskmelon (Cucumis melo L.) is a high-value horticultural crop. The prediction of node appearance is an important part of simulation models of crops with decumbent growth like muskmelon and other cucurbits. One way to predict node appearance is by using the concept of plastochron, defined as the time interval between the appearance of successive nodes on a stem or vine. Time can be expressed as thermal time, and in this case, the plastochron has units of ${ }^{\circ} \mathrm{C}$ day node ${ }^{-1}$. Previous studies have demonstrated that the plastochron in muskmelon varies with genotype and planting date. The objective of this study was to estimate the plastochron in muskmelon grown inside a plastic greenhouse at different transplanting dates. Twelve planting dates were performed

${ }^{1}$ Departamento de Fitotecnia (DF), Centro de Ciências Rurais (CCR), Universidade Federal de Santa Maria (UFSM). 97105-900, Santa Maria, RS, Brasil. E-mail: nstreck1@smail.ufsm.br. Autor para correspondência.

${ }^{2}$ Curso de Graduação em Agronomia, CCR, UFSM, Santa Maria, RS, Brasil.

${ }^{3}$ Centro Universitário Franciscano (UNIFRA) e DF, CCR, UFSM, Santa Maria, RS, Brasil.

${ }^{4} \mathrm{DF}$, CCR, UFSM, Santa Maria, RS, Brasil.

${ }^{5}$ Programa de Pós-graduação em Agronomia, CCR, UFSM, Santa Maria, RS, Brasil. 
inside a $10 \mathrm{~m} X 25 \mathrm{~m}$ greenhouse covered with polyethylene in Santa Maria, RS, Brazil. The HY-MARK hybrid was used. Twelve plants in each planting date were tagged with colored wire. The number of visible nodes $(N N)$ on the main vine of the tagged plants was measured twice a week. Daily degreedays $\left(D D D,{ }^{\circ} \mathrm{C}\right.$ day) were calculated, with cardinal temperatures for node appearance in muskmelon $\left(10,34\right.$, and $\left.45^{\circ} \mathrm{C}\right)$. Accumulated thermal time $\left(T T,{ }^{\circ} \mathrm{C}\right.$ day) from transplanting was calculated by accumulating DDD. The $N N$ was linearly regressed against TT. The plastochron was estimated by the inverse of the angular coefficient of the linear regression. Plastochron varied among planting dates, from 13.4 to $21.8^{\circ} \mathrm{C}$ day node ${ }^{-1}$, with an average of $18.6( \pm 2.3)^{\circ} \mathrm{C}$ day node ${ }^{-1}$. This difference in plastochron values among planting dates may represent several calendar days when $N N$ is used to estimate the date of developmental stages in muskmelon such as flowering of the first male and hermaphrodite flowers.

Key words: temperature, thermal time, node appearance, plant development.

\section{INTRODUÇÃO}

O meloeiro (Cucumis melo L.) é uma cultura de alto valor econômico. O Brasil ocupa o terceiro lugar em produção de melão da América do Sul, depois da Argentina e do Chile, com $17 \%$ da produção total (GRANGEIRO et al., 1999; COSTA, 2001). No Brasil, a área colhida com meloeiro em 2001 foi de 14.198ha com uma produção de aproximadamente 264 milhões de frutos (NAKAMEA, 2004). Na região Nordeste, temse em torno de $90 \%$ da produção total do País. O Estado do Rio Grande do Norte é o maior produtor brasileiro com aproximadamente $42 \%$ da área colhida e 50\% da produção de frutos (NAKAMEA, 2004). O Estado do Rio Grande do Sul é o terceiro maior produtor brasileiro em área colhida e o quarto em produção de frutos (NAKAMEA, 2004).

Por ser uma espécie cujas temperaturas cardinais de crescimento e desenvolvimento são elevadas, o cultivo do meloeiro a campo no Sul do Brasil é restrito aos meses mais quentes do ano (novembro a março). Nos meses de primavera e outono, o seu cultivo tem sido possível somente no interior de estufas plásticas.

O cálculo da taxa de aparecimento de nós na haste principal e em hastes secundárias é um componente importante em modelos matemáticos de simulação do crescimento e desenvolvimento de culturas de hábito de crescimento decumbente como o meloeiro e outras cucurbitáceas (STRECK, 2002). Integrando-se a taxa de aparecimento de nós, tem-se o número total ou acumulado de nós (NN) na haste em um deteminado momento do ciclo de desenvolvimento da planta, o que é uma excelente medida de desenvolvimento vegetal, pois o aparecimento de folhas e flores e a época de ocorrência de outros estágios de desenvolvimento da planta estão relacionados com o NN (HAVELY, 1985; ATHERTON, 1987; STRECK et al., 2003a). No modelo de simulação do crescimento e desenvolvimento do meloeiro proposto por BAKER et al. (2001), a data de abertura da $1^{\text {a }}$ flor masculina e hermafrodita é calculada no dia do aparecimento do $9^{\circ}$ e $12^{\circ}$ nós na haste principal, respectivamente.

A emissão de nós na haste pode ser estimada a partir do conhecimento do tempo necessário para o aparecimento de dois nós sucessivos na planta. Em dicotiledôneas, o intervalo de tempo entre o aparecimento de nós sucessivos na haste é denominado de plastocrono (SINCLAIR, 1984; BAKER \& REDDY, 2001).

Uma unidade de tempo bastante utilizada para representar tempo em plantas é a soma térmica. A soma térmica é uma melhor medida de tempo biológico em plantas do que dias do calendário civil (por exemplo, dia do ano ou dias após a semeadura ou transplante), pois leva em conta o efeito da temperatura sobre o desenvolvimento vegetal, a qual é um dos principais fatores ecológicos que governam o desenvolvimento de plantas, incluindo o aparecimento de nós e folhas (GILMORE \& ROGERS, 1958; RUSSELE et al., 1984; McMASTER \& SMIKA, 1988). O plastocrono, neste caso, é a soma térmica necessária para o aparecimento de um nó na haste da planta e tem como unidade ${ }^{\circ} \mathrm{C}$ dia nó $^{-1}$. O valor do plastocrono é dependente do genótipo de meloeiro e para o mesmo genótipo pode variar com a época de cultivo (BAKER \& REDDY, 2001). Portanto, é importante sua determinação para distintas cultivares e híbridos em diferentes épocas de cultivo.

O presente trabalho teve por objetivo estimar o plastocrono em meloeiro híbrido HY-MARK transplantado em diferentes épocas no interior de estufa plástica.

\section{MATERIAL E MÉTODOS}

O experimento foi conduzido no Campo Experimental do Departamento de Fitotecnia da Universidade Federal de Santa Maria, Santa Maria, RS

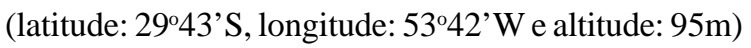
no interior de uma estufa plástica de $10 \mathrm{~m}$ X $25 \mathrm{~m}$ coberta com polietileno transparente de baixa densidade. Foram realizadas 12 épocas de semeadura e transplante durante os anos de 2002, 2003 e 2004 (Tabela 1).

O genótipo usado neste estudo foi o híbrido HY-MARK, do grupo Cantaloupe. A semeadura foi realizada em bandejas de isopor com substrato comercial Plantmax, composto por casca de pinus 
Tabela 1 - Datas de semeadura e transplante, temperatura média do ar no interior da estufa e brilho solar real durante o período usado para estimativa do plastocrono, e valores estimados de plastocrono no meloeiro, híbrido HY-MARK. Santa Maria, RS.

\begin{tabular}{|c|c|c|c|c|c|}
\hline Éроса & Semeadura & Transplante & $\begin{array}{c}\text { Temperatura } \\
\text { média do ar }\left({ }^{\circ} \mathrm{C}\right)\end{array}$ & $\begin{array}{l}\text { Brilho solar } \\
\text { real }\left(\mathrm{h} \mathrm{dia}^{-1}\right)\end{array}$ & $\begin{array}{l}\text { Plastocrono } \\
\left({ }^{\circ} \mathrm{C} \text { dia nó }{ }^{-1}\right)\end{array}$ \\
\hline 1 & 19/10/2002 & $15 / 11 / 2002$ & 22,4 & 6,1 & 13,4 \\
\hline 2 & 19/11/2002 & 20/12/2002 & 25,4 & 8,8 & $18,9 \quad$ с \\
\hline 3 & 20/12/2002 & $14 / 01 / 2003$ & 24,3 & 8,2 & 20,2 bc \\
\hline 4 & 26/02/2003 & 27/03/2003 & 19,8 & 6,2 & 21,8 a \\
\hline 5 & $13 / 08 / 2003$ & $17 / 09 / 2003$ & 20,7 & 6,2 & 16,2 e \\
\hline 6 & 09/10/2003 & $12 / 11 / 2003$ & 23,7 & 7,5 & 17,3 \\
\hline 7 & $17 / 11 / 2003$ & $17 / 12 / 2003$ & 25,7 & 8,4 & 20,0 bc \\
\hline 8 & $26 / 12 / 2003$ & $26 / 01 / 2004$ & 25,6 & 8,6 & $19,0 \quad$ с \\
\hline 9 & $24 / 01 / 2004$ & $20 / 02 / 2004$ & 24,3 & 7,5 & 17,1 de \\
\hline 10 & $13 / 05 / 2004$ & $14 / 07 / 2004$ & 18,0 & 5,7 & 19,6 bc \\
\hline 11 & $21 / 06 / 2004$ & 20/08/2004 & 19,8 & 6,5 & 18,7 cd \\
\hline 12 & 24/08/2004 & 07/10/2004 & 22,0 & 7,9 & $21,3 \mathrm{ab}$ \\
\hline Média ( $\pm \mathrm{DP})$ & & & $22,6( \pm 2,6)$ & $7,3( \pm 1,1)$ & $18,6( \pm 2,3)$ \\
\hline
\end{tabular}

Médias de plastocrono seguidas pela mesma letra na vertical não diferem significativamente entre si pelo teste de Duncan ao nível de 5\% de probabilidade de erro.

triturada, perlita, vermiculita expandida e fertilizantes. O transplante foi realizado quando as plântulas tinham duas folhas definitivas visíveis para sacolas plásticas perfuradas contendo 10 litros com o mesmo substrato comercial acima citado, as quais foram arranjadas em fileiras no interior da estufa no espaçamento de $1 \mathrm{~m}$ entre fileiras e $0,33 \mathrm{~m}$ entre plantas. As plantas foram conduzidas verticalmente e sustentadas com fio de ráfia. Foram feitas podas e desbastes dos ramos laterais semanalmente e deixada apenas uma haste (haste principal) por planta. As plantas foram irrigadas diariamente com auxílio de mangas plásticas gotejadoras de modo a manter a umidade do substrato sempre com água suficiente para garantir a transpiração máxima das plantas, observada empiricamente pela inexistência de sintomas de murchamento folhar. Duas vezes por semana foi realizada a fertilização com solução contendo macro e micro nutrientes, cujas fontes eram vários sais minerais. Os sais minerais e a quantidade usados em cada fertilização do meloeiro foram as seguintes: nitrato de cálcio: 5,69g planta ${ }^{-1}$, cloreto de potássio: 2,00 $\mathrm{g} \mathrm{planta}^{-1}$, sulfato de magnésio: 2,48g planta $^{-1}$, superfosfato simples: $0,87 \mathrm{~g}_{\text {planta }}{ }^{-1}$, uréia: $0,42 \mathrm{~g}_{\text {planta }}{ }^{-1}$, molibdato de sódio: $3,07 \times 10^{-4} \mathrm{~g}$ planta ${ }^{-1}$, ácido bórico: $6,59 \times 10^{-3} \mathrm{~g}_{\text {planta }}{ }^{-1}$, sulfato de cobre: $1,09 \times 10^{-3} \mathrm{~g}_{\text {planta }}{ }^{-1}$, sulfato de manganês: 8,78 x $10^{-3} \mathrm{~g}_{\text {planta }}{ }^{-1}$, sulfato de zinco: $4,39 \times 10^{-3} \mathrm{~g}_{\text {planta }}{ }^{-1}$, quelato de ferro: $8,59 \times 10^{-2} \mathrm{ml} \mathrm{planta}^{-1}$.

A unidade experimental constituiu-se de três fileiras de 5,6m de comprimento (17 plantas por fileira), com as duas fileiras laterais como bordadura e a fileira central como área útil $\left(4,94 \mathrm{~m}^{2}\right)$. O delineamento experimental foi de blocos ao acaso com três repetições. Em cada unidade experimental foram selecionadas quatro plantas logo após o transplante das plântulas, as quais foram etiquetadas com arames coloridos, totalizando 12 plantas etiquetadas por época. Nessas plantas etiquetadas, contou-se o número de nós visíveis na planta duas vezes por semana. Um nó foi considerado como visível quando a folha associada a este nó tinha um comprimento mínimo da maior dimensão da folha de 3cm(BAKER \& REDDY, 2001).

A temperatura do ar foi medida com um termohigrógrafo no interior de um miniabrigo instalado a $1,5 \mathrm{~m}$ de altura no centro da estufa. Dos termohigrogramas foram retirados os dados diários de temperatura mínima e máxima do ar.

A soma térmica diária (STd, ${ }^{\circ} \mathrm{C}$ dia) a partir do transplante foi calculada através das equações (GILMORE \& ROGERS, 1958; ARNOLD, 1960):

$\mathrm{STd}=\left[\left(\mathrm{T}_{\mathrm{ot}}-\mathrm{T}_{\mathrm{b}}\right)\left(\mathrm{T}-\mathrm{T}_{\mathrm{b}}\right) /\left(\mathrm{T}_{\mathrm{ot}}-\mathrm{T}_{\mathrm{b}}\right)\right] .1 \mathrm{dia}$ quando $\mathrm{T}_{\mathrm{b}} \leq \mathrm{T} \leq \mathrm{T}_{\text {ot }}$ e

$\mathrm{STd}=\left[\left(\mathrm{T}_{\mathrm{ot}}-\mathrm{T}_{\mathrm{b}}\right)\left(\mathrm{T}-\mathrm{T}_{\mathrm{up}}\right) /\left(\mathrm{T}_{\mathrm{ot}}-\mathrm{T}_{\mathrm{up}}\right)\right] .1$ dia quando $\mathrm{T}_{\text {ot }} \leq \mathrm{T} \leq \mathrm{T}_{\mathrm{u}}$

em que T é a temperatura média diária do ar no interior da estufa, calculada pela média aritmética da temperatura máxima e mínima diária do ar, $\mathrm{e}_{\mathrm{b}}, \mathrm{T}_{\mathrm{ot}} \mathrm{e}$ $\mathrm{T}_{\text {up }}$ são as temperaturas base, ótima e máxima de aparecimento de nós em meloeiro, consideradas como 10, 34 e $45^{\circ} \mathrm{C}$, respectivamente (BAKER \& REDDY, 2001). A representação gráfica da concepção de cálculo da STd está na figura 1.

A soma térmica acumulada (STa, ${ }^{\circ} \mathrm{C}$ dia) apartir do dia de transplante foi calculada por: 


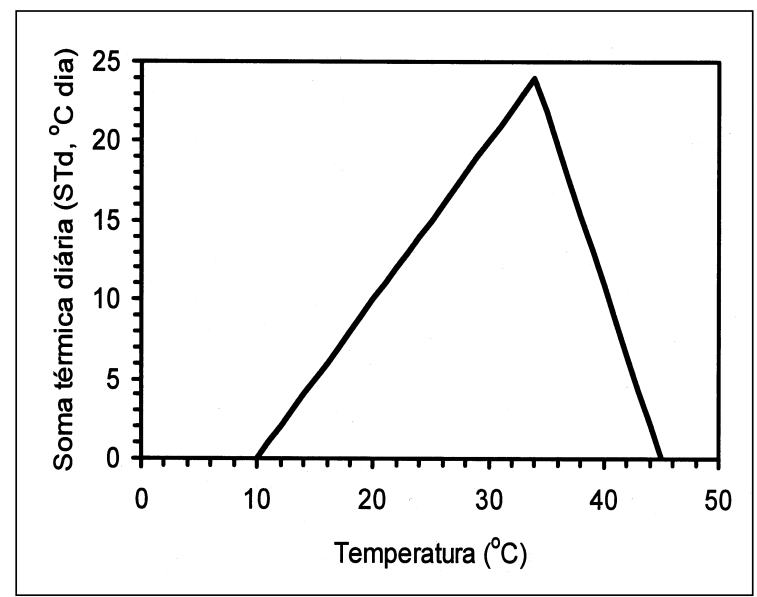

Figura 1 - Método de cálculo da soma térmica diária utilizada no estudo, com o uso das temperaturas cardinais base, ótima e máxima de aparecimento de nós em meloeiro de 10 , 34 e $45^{\circ} \mathrm{C}$, respectivamente. Santa Maria, RS, 2002/2004.

$$
\mathrm{STa}=\sum \mathrm{STd}
$$

Para cada época de cultivo, obteve-se a regressão linear entre o número de nós acumulados (NN) na planta e a soma térmica acumulada (STa) apartir do transplante (SINCLAIR, 1984; BAKER \& REDDY, 2001; SINCLAIR et al., 2005). O plastocrono $\left({ }^{\circ} \mathrm{C}\right.$ dia nó $\left.{ }^{-1}\right)$ foi considerado como sendo o inverso do coeficiente angular da regressão linear entre NN e STa (KLEPPER etal., 1982; KIRBY, 1995; BAKER \& REDDY, 2001). A análise estatística constou da análise da variância e as médias de plastocrono por época foram distinguidas pelo teste de Duncan a 5\% de probabilidade de erro.

\section{RESULTADOS E DISCUSSÃO}

As diferentes épocas de cultivo proporcionaram condições meteorológicas distintas durante o ciclo de desenvolvimento do meloeiro (Tabela 1), pois ocorreram em meses de primavera, verão, outono, e inverno, quando a temperatura do ar e a densidade de fluxo de radiação solar global incidente em Santa Maria são distintas. A exposição a diferentes condições meteorológicas é fundamental nos estudos de avaliação de parâmetros de crescimento e desenvolvimento vegetal e de uso da soma térmica como medida de tempo biológico em plantas.

Valores elevados de correlação entre NN e STa, com um $\mathrm{R}^{2}$ acima de 0,97 , foram verificadas para todas as épocas de cultivo. Um exemplo da relação entre número de nós acumulados na haste principal
(NN) e a soma térmica acumulada (STa) na época 3 está representada na figura 2. Observa-se a relação linear entre $\mathrm{NN}$ e STa, com um coeficiente de determinação $\mathrm{R}^{2}$ de 0,99 . Esta linearidade entre $\mathrm{NN}$ e STa indica que a temperatura do ar é o fator ecológico principal que governa o aparecimento de nós em meloeiro e a estimativa do plastocrono pelo método da regressão linear entre NN e STa é uma metodologia apropriada (SINCLAIR et al., 2005).

Houve diferença estatística de plastocrono entre as épocas de cultivo (Tabela $1)$. Os maiores valores de plastocrono $\left(21,8^{\circ} \mathrm{C}\right.$ dia nó ${ }^{-1}$ e $21,3^{\circ} \mathrm{C}$ dia nó ${ }^{-1}$ ) foram calculados nas épocas 4 (transplante em 27/03/2003) e 12 (transplante em 24/08/2003), respectivamente, os quais não diferiram estatisticamente entre si. O menor valor de plastocrono $\left(13,4^{\circ} \mathrm{C}\right.$ dia nó $\left.{ }^{-1}\right)$ foi calculado na época 1 (transplante em 15/11/2002) e este valor de plastocrono foi estatisticamente diferente de todos os valores nas demais épocas de cultivo.

O valor médio estimado de plastocrono para o meloeiro HY-MARK foi de $18,6( \pm 2,3)^{\circ} \mathrm{C}$ dia nó ${ }^{-1}$ (Tabela 1). Este valor médio de plastocrono é inferior aos valores reportados por BAKER \& REDDY (2001) para as cultivares Explorer $\left(34,8 \pm 1,1^{\circ} \mathrm{C}\right.$ dia nó $\left.{ }^{-1}\right)$, Gold Rush $\left(33,2 \pm 0,9^{\circ} \mathrm{C}\right.$ dia nó $\left.{ }^{-1}\right)$ e Mission $\left(32,9 \pm 1,9^{\circ} \mathrm{C}\right.$ dia nó $\left.^{-1}\right)$. Ressalta-se que diferenças genotípicas são esperadas no que se refere ao plastocrono em meloeiro (BAKER \& REDDY, 2001).

O plastocrono calculado entre as épocas de cultivo variou de 13,4 a $21,8^{\circ} \mathrm{C}$ dia nó ${ }^{-1}$ (Tabela 1 ) ou seja, uma variação de $8,4^{\circ} \mathrm{C}$ dia nó ${ }^{-1}$. Esta variação é similar às diferenças de plastocrono entre épocas de cultivo a campo em Overton, Texas, EUA, para as cultivares de meloeiro Explorer $\left(7,4^{\circ} \mathrm{C}\right.$ dia nó $\left.{ }^{-1}\right)$, Gold Rush $\left(5,0^{\circ} \mathrm{C}\right.$ dia nó $\left.{ }^{-1}\right)$ e Mission $\left(8,1^{\circ} \mathrm{C}\right.$ dia nó $\left.{ }^{-1}\right)$ encontradas por BAKER \& REDDY (2001). Maiores valores de plastocrono para cultivo na primavera foram constatados por BAKER \& REDDY (2001), o que não se verificou no presente estudo, onde o maior valor de plastocrono foi verificado na época 4 (outono, transplante em 27/03/2003, Tabela 1). Estes autores apontaram como possível causa da diferença de plastocrono entre épocas de cultivo, o efeito da cobertura do solo com plástico preto que teria aumentado a temperatura do ar junto às plantas nos cultivos de verão em relação aos cultivos de primavera naquele estudo (BAKER \& REDDY, 2001). No presente estudo, havia plástico opaco branco na fileira de plantas cobrindo as sacolas plásticas com o substrato onde o sistema radicular das plantas estava acondicionado, o que elimina a hipótese de 


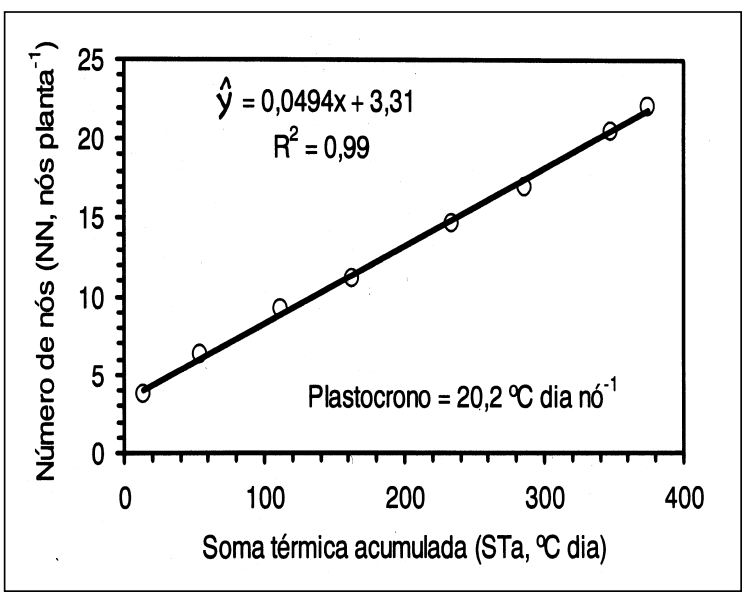

Figura 2 - Relação entre número de nós acumulados na haste principal da planta (NN, nós planta $\left.{ }^{-1}\right)$ e soma térmica acumulada a partir do transplante (STa, ${ }^{\circ} \mathrm{C}$ dia), utilizada para estimativa do plastocrono no meloeiro híbrido HY-MARK, na época de cultivo com transplante em 14/01/2003.

aquecimento diferenciado entre épocas, já que o plástico opaco branco, ao contrário do preto, tem uma alta refletividade à densidade de fluxo de radiação solar incidente e, portanto, é, relativamente, um plástico frio, independente da época do ano.

O fotoperíodo poderia ser uma outra causa para explicar a diferença de plastocrono entre épocas de cultivo. Esta hipótese, no entanto, foi descartada pois BAKER \& REDDY (2001) afirmam não haver registros científicos de que o meloeiro seja sensível ao fotoperíodo e porque, após estes autores terem incluído o fotoperíodo no cálculo da soma térmica, não houve diminuição no coeficiente de variação dos dados de plastocrono nas diferentes épocas de cultivo. No presente estudo, também foi levantada a hipótese de que a diferença de densidade de fluxo de radiação solar incidente entre os meses de verão e inverno em Santa Maria poderia afetar o desenvolvimento do meloeiro, já que a produtividade de pepino, uma espécie similar ao meloeiro em termos de exigências bioclimáticas para crescimento e desenvolvimento, em estufa plástica tem relação com a radiação solar (ZAGO, 2004). Como indicativo da densidade de fluxo de radiação solar incidente nas diferentes épocas de cultivo foi utilizado brilho solar real ( $\mathrm{h} \mathrm{dia}^{-1}$ ) medido na Estação Meteorológica instalada a aproximadamente 100 metros do experimento, pois a densidade de fluxo de radiação solar incidente não foi medida no interior da estufa. No entanto, não houve correlação do plastocrono com este elemento meteorológico $\left(\mathrm{R}^{2}=0,097\right)$, motivo suficiente para descartar também a hipótese de que houvesse efeito da radiação solar sobre o aparecimento de nós em meloeiro. A não existência do efeito da radiação solar sobre o aparecimento de nós no meloeiro está de acordo com resultados anteriores de que este elemento meteorológico não afeta o aparecimento de folhas em trigo (KIRBY, 1995), kiwi (STRECK, 2003) e violeta africana (STRECK, 2004) e reforça o conceito de crescimento e desenvolvimento vegetal como processos independentes, governados por fatores ecológicos distintos (STRECK et al., 2003b).

A diferença de plastocrono de $8,4^{\circ} \mathrm{C}$ dia nó ${ }^{-1}$ entre as épocas de cultivo encontrada neste estudo, é grande. Por exemplo, se o interesse é predizer eventos associados ao NN na planta de meloeiro, como no modelo de BAKER et al. (2001), em que a data de abertura da $1^{\mathrm{a}}$ flor masculina e hermafrodita é calculada quando o $9^{\circ}$ e $12^{\circ}$ nós aparaecem na haste principal, respectivamente, uma diferença de $8,4^{\circ} \mathrm{C}$ dia nó ${ }^{-1}$ no platocrono corresponde a um erro de até 76 a $101^{\circ} \mathrm{C}$ dia, respectivamente, o que pode representar vários dias do calendário civil. Quando o plastocrono é a unidade básica para se predizer a data de aparecimento de nós no final do ciclo de desenvolvimento da planta de meloeiro, este erro pode ser ainda maior. Por exemplo, se uma planta de meloeiro na altura de poda tem 30 nós, como foi na maioria dos cultivos deste estudo e é comum em cultivos comerciais, a diferença entre épocas pode alcançar $252^{\circ} \mathrm{C}$ dia, o que representa um erro de muitos dias do calendário civil, especialmente durante o outono, inverno e primavera, quando a temperatura do ar é baixa.

A soma térmica é um método atrativo e muito utilizado para representar tempo biológico em plantas, por ser um método de cálculo simples e geralmente melhora a predição da data de ocorrência de estágios de desenvolvimento em plantas comparado com dias do calendário civil ou número de dias após a semeadura ou plantio (GILMORE \& ROGERS, 1958; RUSSELE et al., 1984; McMASTER \& SMIKA, 1988). Apesar destas vantagens, o método está aberto a críticas por ter a pressuposição de uma relação linear entre desenvolvimento e temperatura. Esta pressuposição é adequada apenas para uma faixa de temperatura entre a temperatura base e a temperatura ótima. Próximo às temperaturas cardinais, a resposta do desenvolvimento vegetal à temperatura é não linear (SHAYKEWICH, 1995; STRECK, 2002; STRECK et al., 2003a,b). Quando o ciclo da cultura ocorre na época recomendada para o seu cultivo, geralmente as temperaturas se enquadram na faixa de resposta linear de desenvolvimento à temperatura, e, neste caso, a soma térmica funciona bem. No entanto, quando o cultivo é realizado fora da época recomendada, a soma 
térmica nem sempre funciona adequadamente. Portanto, o uso da soma térmica como medida de tempo no plastocrono em meloeiro deve ser feito com cautela.

\section{CONCLUSÃO}

A temperatura do ar é o principal fator ecológico que afeta o aparecimento de nós em meloeiro. O tempo médio necessário para o aparecimento de dois nós sucessivos no meloeiro HY-MARK é em torno de $19^{\circ} \mathrm{C}$ dia. Este tempo médio tem uma variação com a época de cultivo.

\section{AGRADECIMENTOS}

Tibola, bolsista de Iniciação Científica (BIC) da Fundação de Amparo à Pesquisa do Estado do Rio Grande do Sul (FAPERGS). Lago e Heldwein, bolsistas Produtividade em Pesquisa do Conselho Nacional de Desenvolvimento Científico e Tecnológico (CNPq). Zago, bolsista de Mestrado da Coordenação de Aperfeiçoamento de Pessoal de Ensino Superior (CAPES).

\section{REFERÊNCIAS}

ARNOLD, C.Y. Maximum-minimum temperatures as a basis for computing heat units. Proceedings of the American Society for Horticultural Sciences, Boston, v.76, n.1, p.682-692, 1960.

ATHERTON, J.G. Manipulation of flowering. London: Butterworth, 1987. 450p.

BAKER, J.T.; REDDY, V.R. Temperature effects on phenological development and yield of muskmelon. Annals of Botany, Oxford, v.87, p.605-613, 2001.

BAKER, J.T. et al. A simple phenological model of muskmelon development. Annals of Botany, Oxford, v.87, p.615-621, 2001.

COSTA, N.D. A cultura do melão. Brasília: Embrapa, Comunicação para Transferência de Tecnologia, 2001. 114p. (Coleção Plantar, 44).

GILMORE, E.C. Jr.; ROGERS, J.S. Heat units as a method of measuring maturity in corn. Agronomy Journal, Madison, v.50, n.10, p.611-615, 1958.

GRANGEIRO, L.C. et al. Rendimento de híbridos de melão amarelo em diferentes densidades de plantio. Horticultura Brasileira, Brasília, v.17, n.3, p.200-206, 1999.

HAVELY, A.H. Handbook of flowering. Boca Raton: CRC, 1985. V. I a V.
KIRBY, E.J. Factors affecting rate of leaf emergence in barley and wheat. Crop Science, Madison, v.35, n.1, p.11-19, 1995.

KLEPPER, B. et al. Quantitative characterization of vegetative development in small cereal grains. Agronomy Journal, Madison, v.7, p.780-792, 1982.

McMASTER, G.S.; SMIKA, D.E. Estimation and evaluation of winter wheat phenology in the central Great Plains. Agricultural and Forest Meteorology, Amsterdam, v.43, n.1, p.1-18, 1988.

NAKAMEA, I.J. Agrianual - anuário da agricultura brasileira. São Paulo: FNP Consultoria e Agroinformativos, 2004. 496p.

RUSSELE, M.P. et al. Growth analysis based on degree days. Crop Science, Madison, v.24, n.1, p.28-32, 1984.

SINCLAIR, T.R. Leaf area development in field grown soybean. Agronomy Journal, Madison, v.76, n.1, p.141146, 1984.

SINCLAIR, T.R. et al. Comparison of vegetative development in soybean cultivars for low-latitude environments. Field Crops Research, Amsterdam, v.92, n.1, p.53-59, 2005.

SHAYKEWICH, C.F. An appraisal of cereal crop phenology modeling. Canadian Journal of Plant Science, Ottawa, v.75, n.2, p.329-341, 1995.

STRECK, N.A. A generalized nonlinear air temperature response function for node appearance rate in muskmelon (Cucumis melo L.). Revista Brasileira de Agrometeorologia, Santa Maria, v.10, n.1, p.105-111, 2002.

STRECK, N.A. A generalized nonlinear temperature response function for some growth and developmantal parameters in kiwifruit (Actinidia deliciosa (A. Chev.) C.F. Liang \& A.R. Ferguson). Ciência Rural, Santa Maria, v.33, n.2, p.255-262, 2003.

STRECK, N.A. A temperature response function for modeling leaf growth and development of the African violet (Saintpaulia ionantha Wendl.). Ciência Rural, Santa Maria, v.34, n.1, p.55-62, 2004

STRECK, N.A. et al. Incorporating a chronology response function into the prediction of leaf appearance rate in winter wheat. Annals of Botany, Oxford, v.92, n.2, p.181-190, 2003a.

STRECK, N.A. et al. Improving predictions of developmental stages in winter wheat: a modified Wang and Engel model. Agricultural and Florest Meteorology, Amsterdam, n.115, p.139-150, 2003b.

ZAGO, V. Influência da radiação solar e da temperatura do ar na produção de pepino em estufa plástica. 2004. 78f. Dissertação (Mestrado em Agronomia) - Programa de Pósgraduação em Agronomia, Universidade Federal de Santa Maria. 